\title{
Light distribution through advanced fenestration systems
}

\author{
Marilyne Andersen \\ Solar Energy and Building Physics Laboratory (LESO-PB), \\ Swiss Federal Institute of Technology (EPFL), CH - 1015 Lausanne, Switzerland. \\ Tel.: +41-21-693-45-51; Fax : +41-21-693-27-22; e-mail: marilyne.andersen@epfl.ch
}

\begin{abstract}
Most energy-saving applications of advanced fenestration systems, e.g. solar blinds, novel types of glazing and daylight redirecting devices, require a precise knowledge of their directional light transmission features. These photometric properties are described by a Bi-directional Transmission Distribution Function (BTDF), which is experimentally assessed by a bi-directional photogoniometer.

As such a function represents a heavy amount of data, there is a need for a synthetic and intuitive visualization of a system's transmission behaviour. For this purpose, four kinds of graphical representations have been created, and are presented in this paper. They are based on bi-directional data assessed by a novel digital imaging-based photogoniometer, whose measurement principle allows a continuous knowledge of the whole transmission space, and therefore an appreciable liberty in data processing.

The geometric properties of the different representations are described, together with the corresponding image operations. The information extraction from these graphical visualizations is given through a comparison example between a conventional venetian blind and an optimized prototype.
\end{abstract}

Keywords: advanced windows, Bi-directional Transmission Distribution Function (BTDF), daylighting, digital imaging (CCD), energy, façades, light distribution, photometry, transmission, visual comfort

\section{Introduction}

Efficient collection or redistribution of direct sunlight for optimal visual and thermal comfort conditions in buildings remains a major objective of fenestration systems. They can improve the penetration of daylight into deep rooms to reduce electricity consumption and lead to larger solar gains in winter combined with lower solar loads in summer, which also significantly increases energy savings.

Advanced windows, including novel solar blinds, new glazing materials and daylightredirecting devices of course play an essential role in this field (Wirth, 1999). A complete and precise knowledge of their directional photometric properties is therefore indispensable in order to control the daylighting performances of buildings: the judicious combination of glazing materials, which would be appropriate to a given lighting situation and aiming at better visual comfort and energy savings, can thus be defined already at the project's level (Scartezzini and Paule, 1994). The required information is provided by the Bi-directional Transmission Distribution Function (BTDF), also called Luminance Coefficient q [ $\mathrm{sr}^{-1}$ ] in the CIE nomenclature (1977), and is intended to be used by the building industry: to optimize the luminous performance of innovative solutions for windows on one hand, to facilitate the choice of industrial products (solar blinds, novel kinds of glazing etc.) during the building construction process on the other hand, and to describe photometric properties of complex fenestration and glazing materials according to a common format. Moreover, daylighting simulation programmes also require such detailed transmission data in order to achieve a reliable modelling of light propagation into rooms using advanced fenestration systems.

The BTDF is defined by equation (1), and the associated photometric and geometric quantities are illustrated in Fig. 1:

$$
\operatorname{BTDF}\left(\theta_{1}, \phi_{1}, \theta_{2}, \phi_{2}\right)=\frac{L_{2}\left(\theta_{1}, \phi_{1}, \theta_{2}, \phi_{2}\right)}{L_{1}\left(\theta_{1}, \phi_{1}\right) \cos \theta_{1} d \omega_{1}}=\frac{L_{2}\left(\theta_{1}, \phi_{1}, \theta_{2}, \phi_{2}\right)}{E_{1}\left(\theta_{1}\right)}\left[\frac{c d}{m^{2} l x}\right]
$$


where

$\left(\theta_{1}, \phi_{1}\right)$ are the polar coordinates of the incoming light flux [ $\left.{ }^{\circ}\right]$

$\left(\theta_{2}, \phi_{2}\right)$ are the polar coordinates of the emerging (transmitted) light flux [ $\left.{ }^{\circ}\right]$

$\mathrm{L}_{1}\left(\theta_{1}, \phi_{1}\right)$ is the luminance of the incoming element of light flux [ $\mathrm{cd} \cdot \mathrm{m}^{-2}$ ]

$\mathrm{L}_{2}\left(\theta_{1}, \phi_{1}, \theta_{2}, \phi_{2}\right)$ is the luminance of the emerging (transmitted) element of light flux [cd $\cdot \mathrm{m}^{-2}$ ] $\mathrm{d} \omega_{1}$ is the solid angle subtended by the incoming light flux [sr]

$\mathrm{E}_{1}\left(\theta_{1}\right)$ is the illuminance of the fenestration material, due to the incoming light flux [lx].

The corresponding beam is generally collimated, showing a constant illuminance $\mathrm{E}_{1}\left(\theta_{1}\right)$ on the section of the characterized sample.

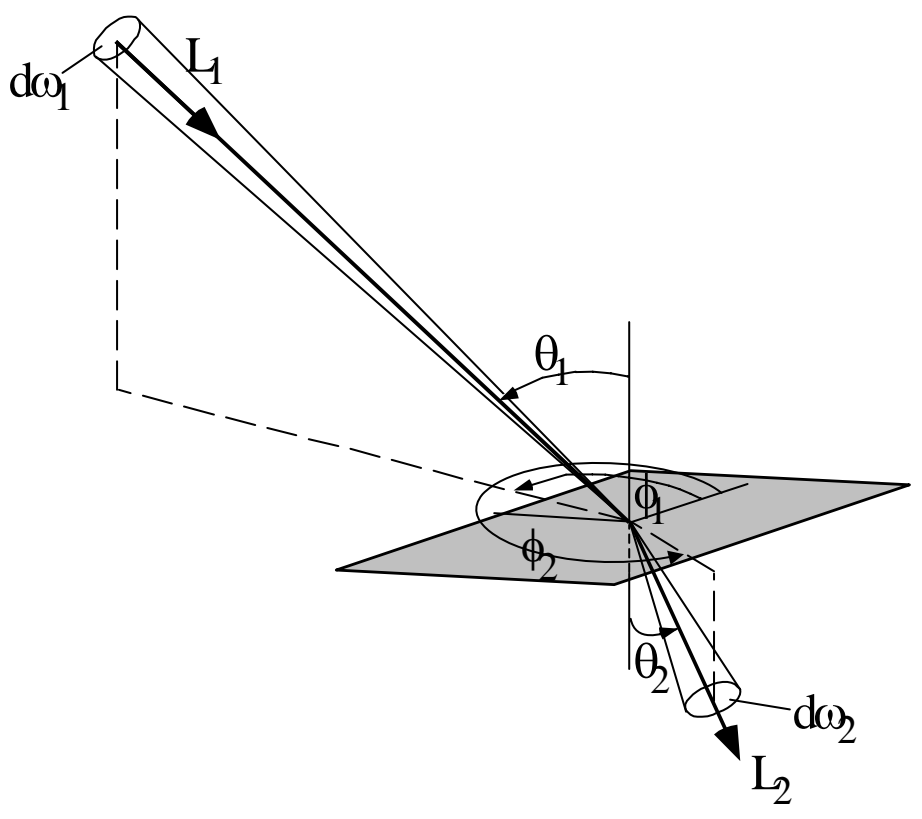

Fig. 1. Photometric and geometric quantities used to define the Bi-directional Transmission Distribution Function (BTDF) of a fenestration material.

The second BTDF formulation of equation (1) is closer to the CIE (1977) nomenclature, which defines the luminance coefficient $\mathrm{q}\left[\mathrm{cd}^{-2} \mathrm{~m}^{-2} \cdot \mathrm{lx}^{-1}\right]$ as "the quotient of the luminance of a surface element in a given direction, by the illuminance incident on the sample".

This definition allows a very easy and direct application of numeric values of BTDFs to practical cases. Indeed, to know how much light (i.e. what luminance $\mathrm{L}_{2}$ ) comes out from a certain area of the sample along a particular direction $\left(\theta_{2}, \phi_{2}\right)$ (and with a particular incident direction of the sun $\left.\left(\theta_{1}, \phi_{1}\right)\right)$, one only needs to multiply the known BTDF value by a realistic illuminance, $\mathrm{E}_{1}$, applicable in the studied situation:

$$
L_{2}\left(\theta_{1}, \phi_{1}, \theta_{2}, \phi_{2}\right)=\operatorname{BTDF}\left(\theta_{1}, \phi_{1}, \theta_{2}, \phi_{2}\right) \cdot E_{1}\left(\theta_{1}\right)
$$

As a BTDF depends on four different parameters $\theta_{1}, \phi_{1}, \theta_{2}$ and $\phi_{2}$ - gathered in files, one for each incident direction $\left(\theta_{1}, \phi_{1}\right)$-, the amount of numerical data becomes very large and cannot offer a synthetic view of the sample's transmission features. A graphical processing is therefore necessary to provide a reasonable appreciation of the light-transmission behaviour: three-dimensional graphical representations of BTDFs have thus been created to provide intuitive visualization possibilities of the light redistribution, which are described here. As the data have been assessed by a digital imaging-based bi-directional photogoniometer, developed at the LESO-PB, EPFL (Andersen et al., 2001), a particular image processing has been developed as well to take the benefit from a continuous information about the transmitted light distribution, moreover provided at a very high spatial accuracy (pixel level). 


\section{Coordinate system}

The characterization of a daylighting system with respect to different incident and emerging angles of the light flux requires the definition of a coordinate system, preferably based on international agreements. Within the framework of Task 21 of the International Energy Agency, a common format was determined (Aydinli, 1999).

The origin of the coordinate system is placed on the daylighting component. Directions are defined by spherical coordinates, which is the most intuitive representation of lighttransmission behaviour: altitude angle $\theta_{\mathrm{i}}$, which is between $0^{\circ}$ and $90^{\circ}$, and azimuth angle $\phi_{\mathrm{i}}$, which is between $0^{\circ}$ and $360^{\circ}$ (Fig. 1). Index $\mathrm{i}$ indicates whether it is related to the incident $(i=1)$ or transmitted $(i=2)$ direction..

The relative position of a component to the coordinate system hugely impacts to the measurement results. Accordingly, the orientation of the sample must also be precisely defined to make BTDF measurement comparisons possible. The following rules (Fig. 2) apply to the adjustment:

- The sample plane is parallel to a vertical window plane. The origin axes $\theta_{1}=0^{\circ}$ and $\theta_{2}=$ $0^{\circ}$, orthogonal to the sample element, point horizontally towards outside and inside the building respectively.

- Placing the component on a vertical façade and observing it from outside, the $\phi_{\mathrm{i}}=0^{\circ}$ axes point horizontally towards the right hand side; axes $\phi_{\mathrm{i}}=90^{\circ}$ therefore point towards the top. Besides, in case of samples presenting parallel features (slats, gratings), the indicated direction will correspond to $\phi_{\mathrm{i}}=0^{\circ}$.

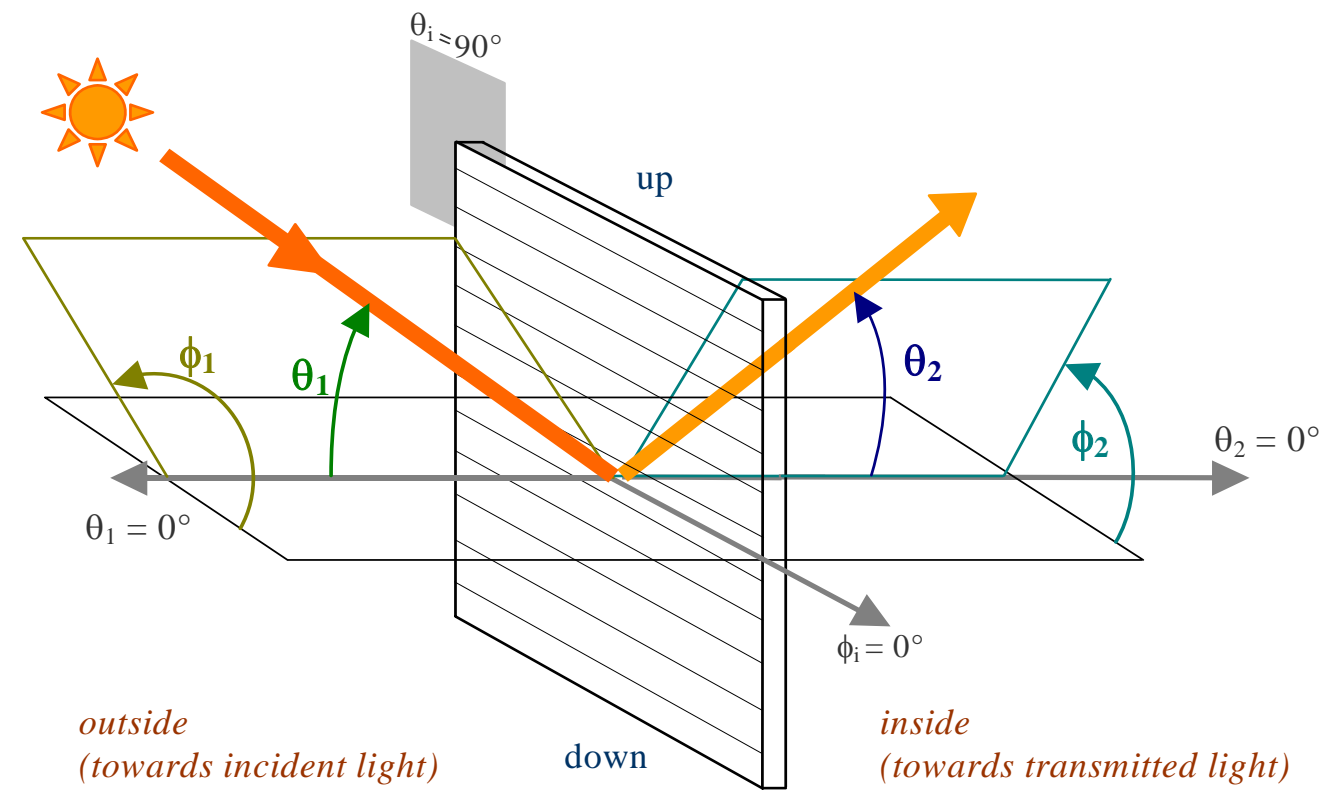

Fig. 2. Orientation of a daylighting component with regard to the coordinate system. Input angles are indexed by 1 , output angles by 2 .

\section{Image and data processing}

The functioning principle of the bi-directional photogoniometer developed at the LESO-PB, EPFL (Andersen et al., 2001) and based on digital imaging techniques is the following: the intuitive approach consisting of an observation of the transmitted light on a hemispherical surface with a mobile sensor (point-per-point measurements) is replaced by a projection of 
the transmitted light on a diffusing triangular panel (Fig. 3). The latter reflects the light towards a charge-coupled device (CCD) camera, which provides a picture of the whole screen. After six $60^{\circ}$ rotations of this system, with image capture and calibration at each position, the transmitted light distribution is fully known.

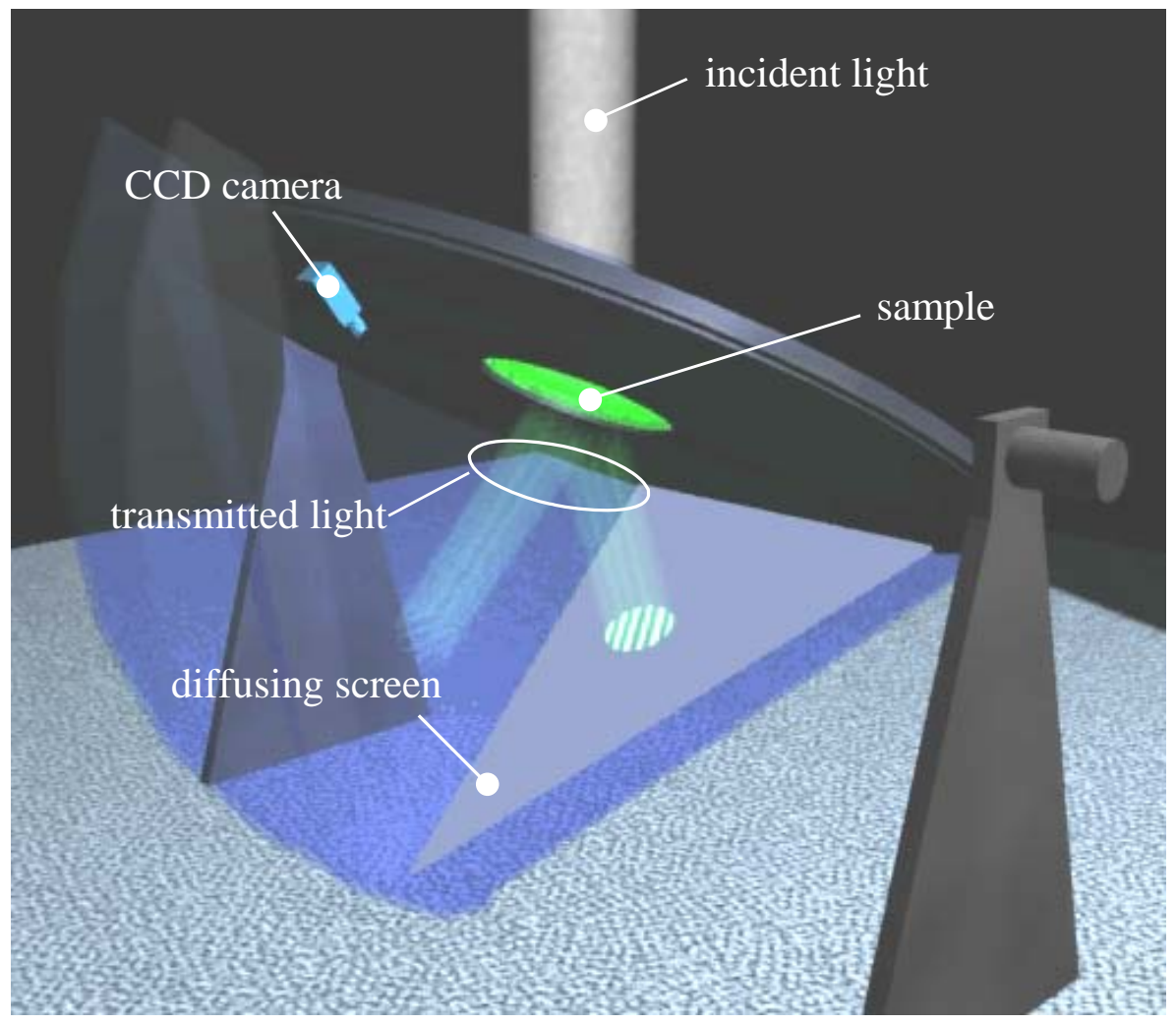

Fig. 3. Functioning principle of the LESO-PB bi-directional photogoniometer using a CCD camera as a multiple-points luminance-meter.

At each screen position, picture snapshots are taken with several integration times, and grey levels are transformed into associated luminances. The superposition of different exposures for the same luminous situation allows one to improve the accuracy of luminance measurements and to avoid over- and/or under-exposure in presence of high luminance dynamics. The obtained "screen" luminance values are finally converted into BTDF data, where distance and light tilting effects are compensated.

This digital imaging-based measurement facility and its calibration procedures are presented in details in Andersen et al. (2001), together with the image and data-processing procedures. A more thorough investigation of the results validation can be found in Andersen et al. (2000).

In order to obtain BTDFs according to a regular output resolution, e.g. every $10^{\circ}$ in altitude and $15^{\circ}$ in azimuth, outgoing zones have to be defined around the considered directions (e.g. corresponding to intervals $\theta_{2} \pm 5^{\circ}, \phi_{2} \pm 7.5^{\circ}$ ). BTDF data are thus provided as averages over given outgoing zones, and the whole transmission space is investigated without any unexplored area.

Such a discretization of the output hemisphere into zones representing average light emergence around particular directions $\left(\theta_{2}, \phi_{2}\right)$ has the important advantage of providing a continuous characterization of the light distribution. In addition to great time-consumption reduction and to accuracy upholding even for high luminance dynamics, the use of digital 
imaging for photogoniometric measurements stops the loss of information on the system's transmission properties, unlike point-per-point investigations where interpolation is needed for the in-between regions, and where the risk of missing a narrow transmission feature is never zero.

Three kinds of outputs are provided after a sample characterization is performed by this digital image-based facility: a BTDF data file, a recomposed visualization of the transmission with the CCD camera, and three graphical representations of the data.

\subsection{Integral data set}

The achieved BTDF data set is saved in ASCII format on an electronic file denominated after the sample name, and including the institute's designation and the considered incident direction (e.g. leso_SampleName_ $\theta_{1} \_\phi_{1}$.txt). Numerical BTDF values can be used to analyse the sample's adequacy for the visual comfort conditions, for instance by comparing the measured values with the reference ranges accounting for glare effects or high luminance contrasts.

This file contains the following data: sample characteristics (manufacturer, symmetry indicator, thickness, etc.), the measurement parameters (incident direction, output angular resolution $\left(\Delta \theta_{2}, \Delta \phi_{2}\right)$, etc.), the hemispherical light transmittance $\tau\left(\theta_{1}, \phi_{1}\right)$ calculated through an integration of the bi-directional data, and the BTDF values, expressed in $\left[\mathrm{cd}^{\cdot} \mathrm{m}^{-2} \cdot \mathrm{lx}^{-1}\right]$, for each associated angular direction $\left(\theta_{2}, \phi_{2}\right)$.

As a result of image processing, the achieved data represent average values of the BTDFs measured in the output hemisphere regions limited by $\left(\phi_{2}-1 / 2 \Delta \phi_{2} ; \phi_{2}+1 / 2 \Delta \phi_{2}\right)$ in azimuth and by $\left(\theta_{2}-1 / 2 \Delta \theta_{2} ; \theta_{2}+1 / 2 \Delta \theta_{2}\right)$ in altitude for each outgoing direction, as mentioned above.

\subsection{Complete visualization of the digital images}

The six calibrated images created for the six screen positions are superposed to build up an integral image of the directional transmission represented in polar coordinates, thanks to an image acquisition and processing software called IMAGE-Pro PLus ${ }^{\text {TM }}$, v. 3.0.01.00 (Media Cybernetics, L.P.).

Before being recombined, each individual image requires several corrections in order to compensate for distance and light-tilting effects; as they cannot be applied analytically as for the numerical data, equivalent operations are effectuated on the different images using specific digital treatments.

The six final pictures are then resized (to produce equilateral triangles), rotated (according to the considered screen position) and appropriately positioned on an integral view. These operations are shown in Fig. 4: a white screen is chosen to illustrate the applied procedures. It can be noted that the original screen image is composed of $488 \times 685$ pixels, whereas the final hemispherical image is smaller, in order to reduce the amount of required memory: its size is $400 \times 400$ pixels.

As a consequence of image construction, the final view is not exactly a visualization of the "transmission hemisphere" projected on a horizontal plane; the angular corrections, required to build such a projection, have not been applied on the pictures, the orthogonal projection being too heavy for the image-handling procedures.

Nevertheless, the obtained images offer direct information about the directional transmission of the analysed sample. The possibility to observe some details about transmission features, that could have been rendered invisible because they were averaged inside the different discretization zones can be obtained that way. 

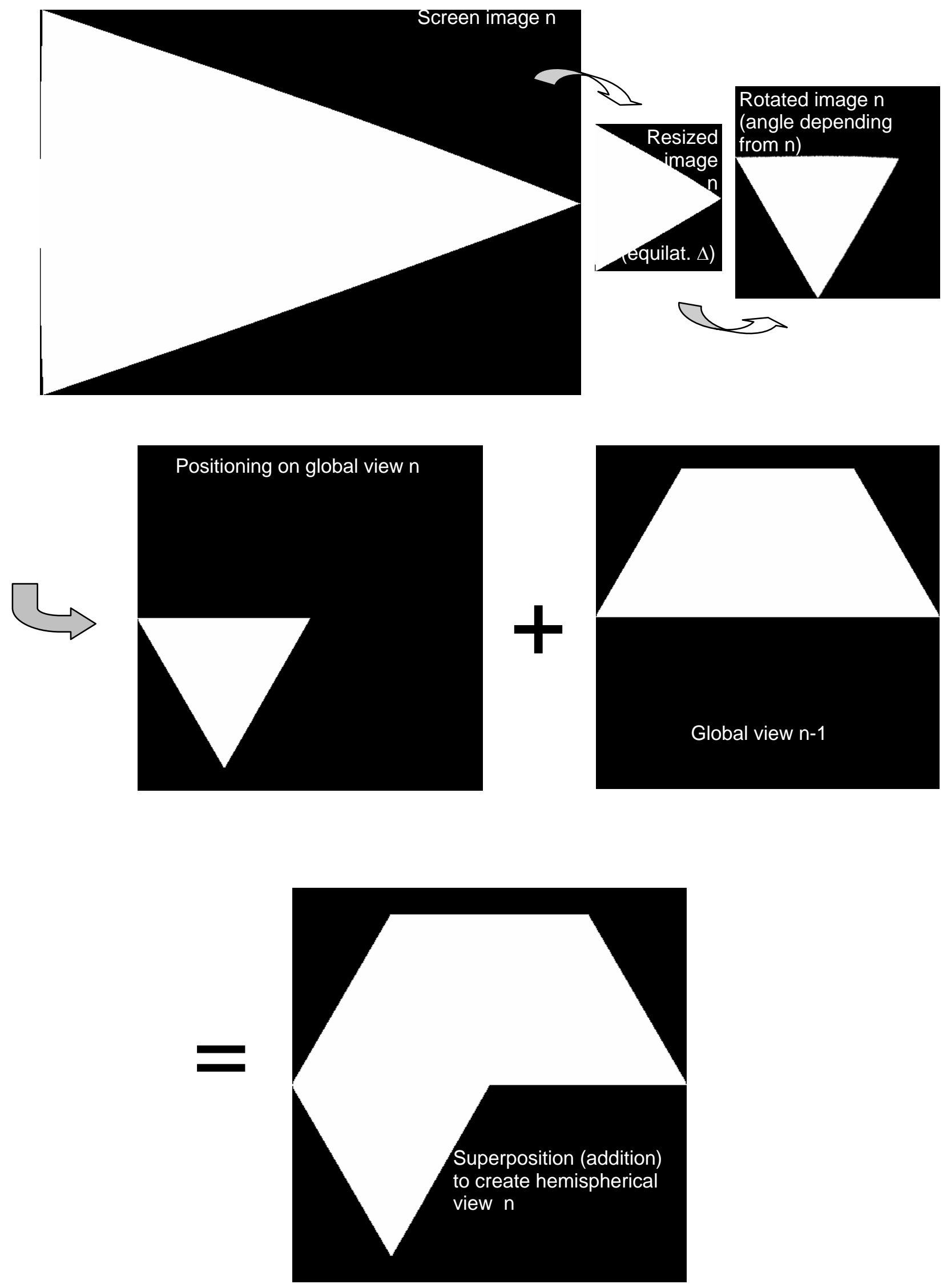

Fig. 4. Operations carried out on each screen image (screen positions $n=1-6$ ) to produce a hemispherical view of the BTDF. The actual position represented here is $n=4$. 
Two examples of hemispherical visualizations are shown in Fig. 5 for a 3M prismatic film under an incidence of $\left(48^{\circ}, 90^{\circ}\right)$ and for a pleated tissue blind (prototype "PLISSEE 3141"), manufactured by Baumann-Hüppe AG and characterized here under normal incidence. To work out what kind of angles the transmission features observed on the image correspond to, a polar grid is drawn to show approximately the values of $\theta_{2}$ and $\phi_{2}$, with $10^{\circ}$ and $15^{\circ}$ steps, respectively. One can immediately observe that the prismatic film presents sharp transmission features, with two main peaks of unequal intensity (brightness), whereas the tissue blind shows a quite diffuse behaviour (similar shade of grey over a large area), even though a brighter part can be outlined.
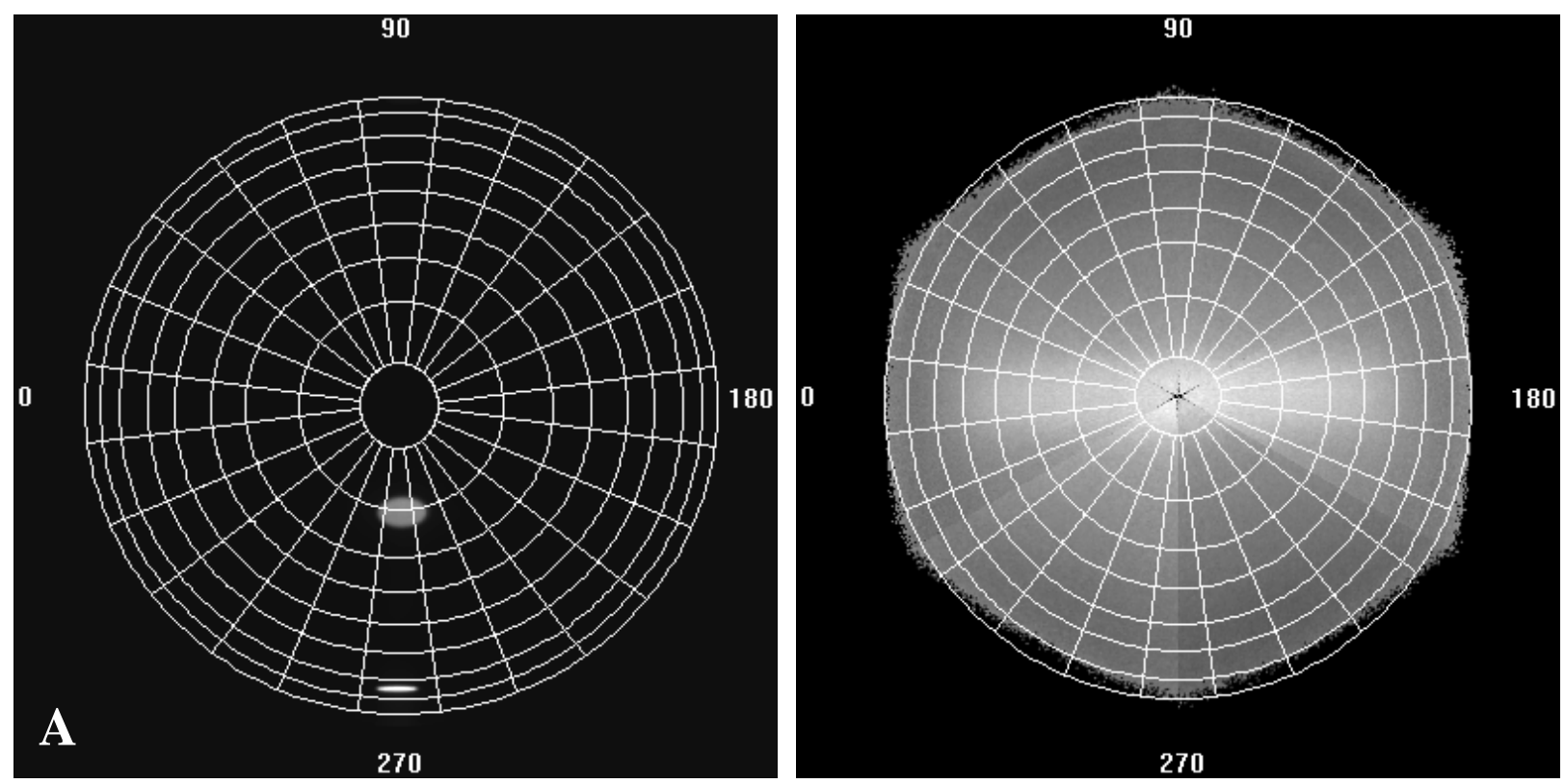

Fig. 5. BTDF hemispherical view: (A) Prismatic SOLF film (3M) : light incidence on flat glass side; $\left(\theta_{1}, \phi_{1}\right)=$ $\left(48^{\circ}, 90^{\circ}\right)$; (B) Pleated tissue blind (Baumann-Hüppe AG) $:\left(\theta_{1}, \phi_{1}\right)=\left(0^{\circ}, 0^{\circ}\right)$.

A particular graphical processing has then been elaborated to create photometric solids from the achieved bi-directional transmission data. The different representations are described below.

\section{Representation of photometric solids}

The set of BTDF numerical data is very large and does not offer a synthetic view of the sample's transmission features. Graphical processing is therefore necessary to provide a reasonable appreciation of the light transmission behaviour. The data are treated for this purpose by MATLAB®, v. 5.3.1 (The MathWorks, Inc.) to create three-dimensional graphical representations of BTDFs; different visualization possibilities of the transmission features are shown in Figs. 6-8 for the prismatic film and the tissue blind presented above under the same incident directions. These representations correspond to an intuitive approach of the light distribution. Moreover, they provide a global point of view of the transmission properties (diffuse, specular, etc.), together with a direct way to pick out its particularities (specular component whereas globally diffusing, apparition of transmission peaks or troughs, etc.). 


\subsection{Hemispherical projection}

The projection of the transmission features on a full hemisphere is based on the following principle: each BTDF value, measured inside a given angular discretization zone, is represented by the corresponding patch standing out against the hemisphere vault. The scale reproduces the BTDF dynamic range for a given incident direction, and allows one to visualize the numerical BTDF data. The point of view under which the hemisphere is seen can be freely chosen by simple mouse dragging and clicking. Fig. 6 shows this projection for the two previously mentioned products, with different output angular resolutions: $\left(\Delta \theta_{2}, \Delta \phi_{2}\right)$ is equal to $\left(5^{\circ}, 5^{\circ}\right)$ for the prismatic film and to $\left(10^{\circ}, 15^{\circ}\right)$ for the solar blind, where wider discretization zones can be observed.

This representation leads to a clear understanding of the angular distribution of the transmitted light flux. The values of $\theta_{2}$ and $\phi_{2}$ can indeed be easily read on this projection.

It must be mentioned that the hemispherical projection is very similar to the visualization offered by the integral calibrated image (Fig. 5), when the viewing direction is vertical. These two representations indeed complement each other: the recomposed image provides details about finer transmission features than the output resolution can render, whereas the hemispherical projection offers a possibility of quantitative evaluation of BTDFs and a more precise angular and spatial management.
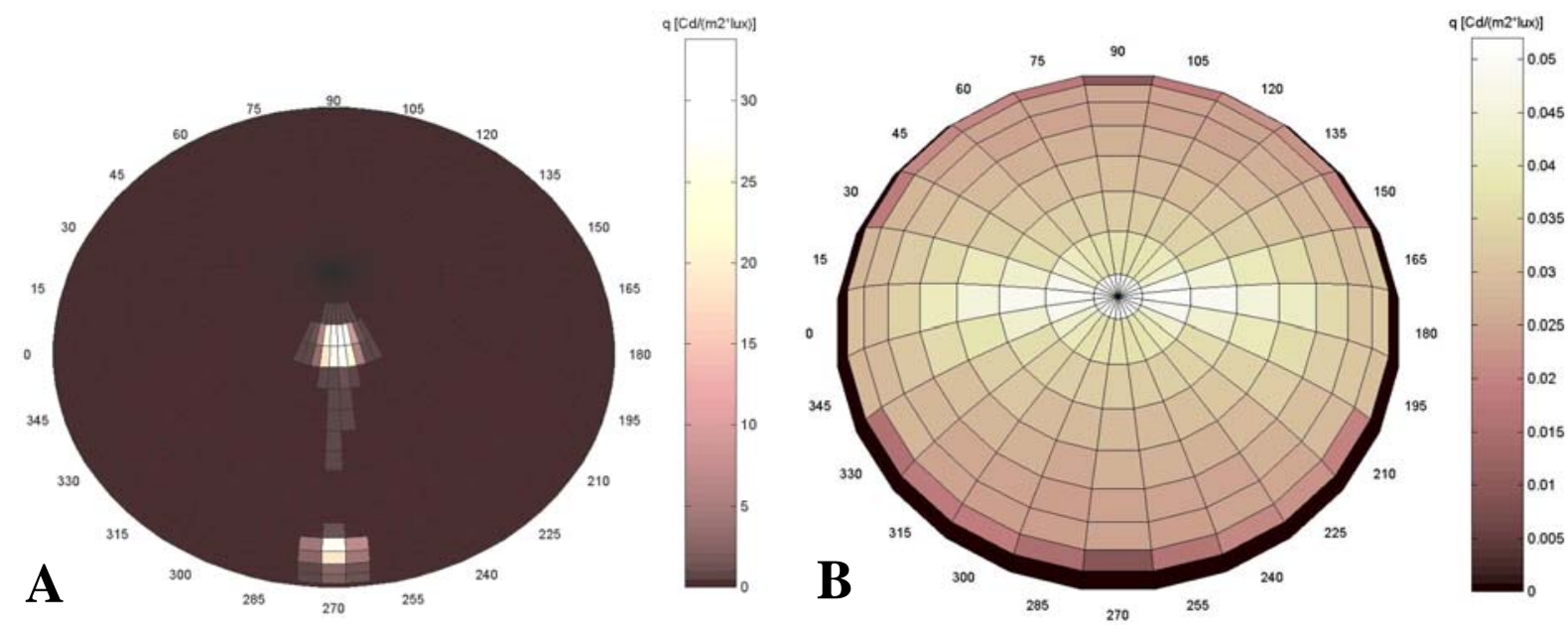

Fig. 6. Hemispherical projections: (A) Prismatic SOLF film (3M) : light incidence on flat glass side; $\left(\theta_{1}, \phi_{1}\right)=$ $\left(48^{\circ}, 90^{\circ}\right) ;\left(\Delta \theta_{2}, \Delta \phi_{2}\right)=\left(5^{\circ}, 5^{\circ}\right)$; (B) Pleated textile solar blind (Baumann-Hüppe AG) : $\left(\theta_{1}, \phi_{1}\right)=\left(0^{\circ}, 0^{\circ}\right) ;\left(\Delta \theta_{2}\right.$, $\left.\Delta \phi_{2}\right)=\left(10^{\circ}, 15^{\circ}\right)$.

\subsection{Photometric solid}

The photometric solid can be defined as a three-dimensional representation of the luminous intensity characteristics, commonly given for artificial lighting fixtures. These solids are also represented by section views of the spatial intensity distribution; such section views can be used for BTDF measurements as well (see below).

Note that the main difference between characterizing luminaires and assessing the performances of daylight systems (or materials, generally speaking) - apart from distinct measured quantities is that the incident direction of the impinging light flux is not a variable parameter for lighting fixtures, which reduces the number of light emergence distributions to one.

The creation of photometric solids requires one to build a grid based on a spherical referential, where each point is represented by a triplet $\left(\mathrm{BTDF}_{\mathrm{val}}, \theta_{2}, \phi_{2}\right), \mathrm{BTDF}_{\mathrm{val}}$ being the numerical value of $\operatorname{BTDF}\left(\theta_{1}, \phi_{1}, \theta_{2}, \phi_{2}\right)$, which is used as the radial distance. The point of view is freely chosen, which allows the observation of every characteristic on the transmission distribution function. 
This graphical representation, therefore, gives a synthetic and intuitive idea of the angular distribution of the transmitted light: peaks in transmission will, for instance, appear like sharp emerging zones, whereas a diffuse transmission will produce a "smooth solid", that looks like a hemisphere and presents only small shade differences. This clear-sighted visualization of the material's transmission features allows a good understanding of its photometric performances as a fenestration material.

To visualize the situation better, the incident direction is represented in the opposite hemisphere as well, through its angular direction $\left(\theta_{1}, \phi_{1}\right)$; a grid representing the polar coordinates $\left(\theta_{2}, \phi_{2}\right)$ is projected on a horizontal plane to clarify the three-dimensional perception of the photometric solid and to allow a direct spatial localisation of particular transmission features (especially when choosing a vertical direction of view).

The scale is proportional to the BTDF range for a given incident direction. This leads to a doublecheck possibility of the BTDF values, through the growing radial distance on one hand, and through a brightening of the associated shade on the other hand, for increasing BTDF values.

Two photometric solids are given in Fig. 7. With these examples, one can easily observe a splitting of the incident beam into two distinct outgoing directions for the prismatic film, which do not correspond to the extension of the incident direction, and a privileged direction for transmission along the vertical plane $0^{\circ}-180^{\circ}$ for the tissue blind (caused by the pleats), combined to a rather diffuse transmittance for the other directions.

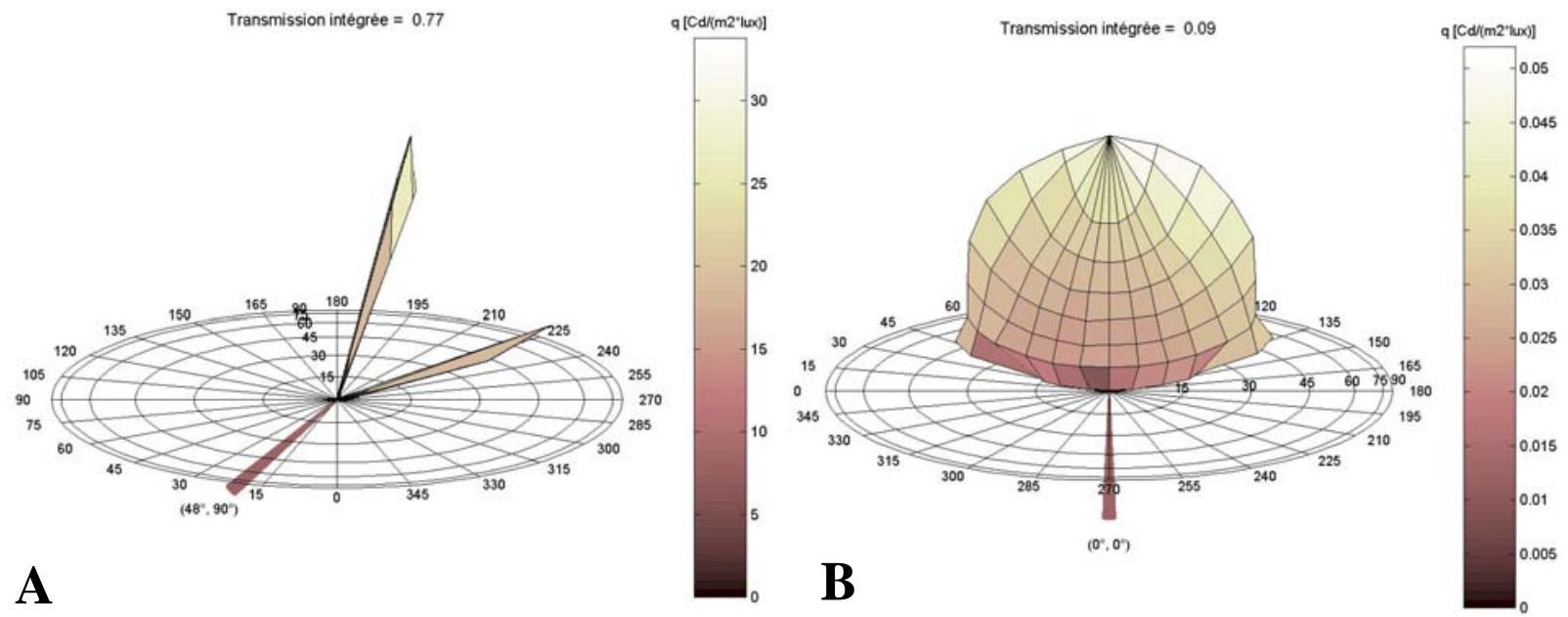

Fig. 7. Photometric solids: (A) Prismatic SOLF film (3M) : light incidence on flat glass side; $\left(\theta_{1}, \phi_{1}\right)=\left(48^{\circ}\right.$, $\left.90^{\circ}\right) ;\left(\Delta \theta_{2}, \Delta \phi_{2}\right)=\left(5^{\circ}, 5^{\circ}\right)$; (B) Pleated textile solar blind (Baumann-Hüppe AG) : $\left(\theta_{1}, \phi_{1}\right)=\left(0^{\circ}, 0^{\circ}\right) ;\left(\Delta \theta_{2}, \Delta \phi_{2}\right)=$ $\left(10^{\circ}, 15^{\circ}\right)$

\subsection{Section views}

The third possible representation consists of several section views of the previously described photometric solid, along vertical planes (C planes perpendicular to the sample plane). A planar curve is provided every $15^{\circ}$ in azimuth (i.e. along planes C0, C15, C30, C45, C60, C75, C90, C105, C120, C135, C150, C165), but this selection can be modified according to specific needs of the user or to particular transmission features (e.g. narrow peaks) that would be more easily observed along other planes. As mentioned before, the obtained curves are similar to the luminous intensity distributions found in artificial lighting catalogues, even though the latter only provide one or two sections in general.

Each curve is showed on a polar coordinates grid, giving the azimuth value of the associated section planes, the BTDF scale and the altitude angles $\theta_{2}$. 
Fig. 8 gives section views of the BTDF distribution for the prismatic film and the solar blind. This representation yields a clear quantitative analysis of the BTDF behaviour, by providing "easy-to-read" and accurate numerical data.
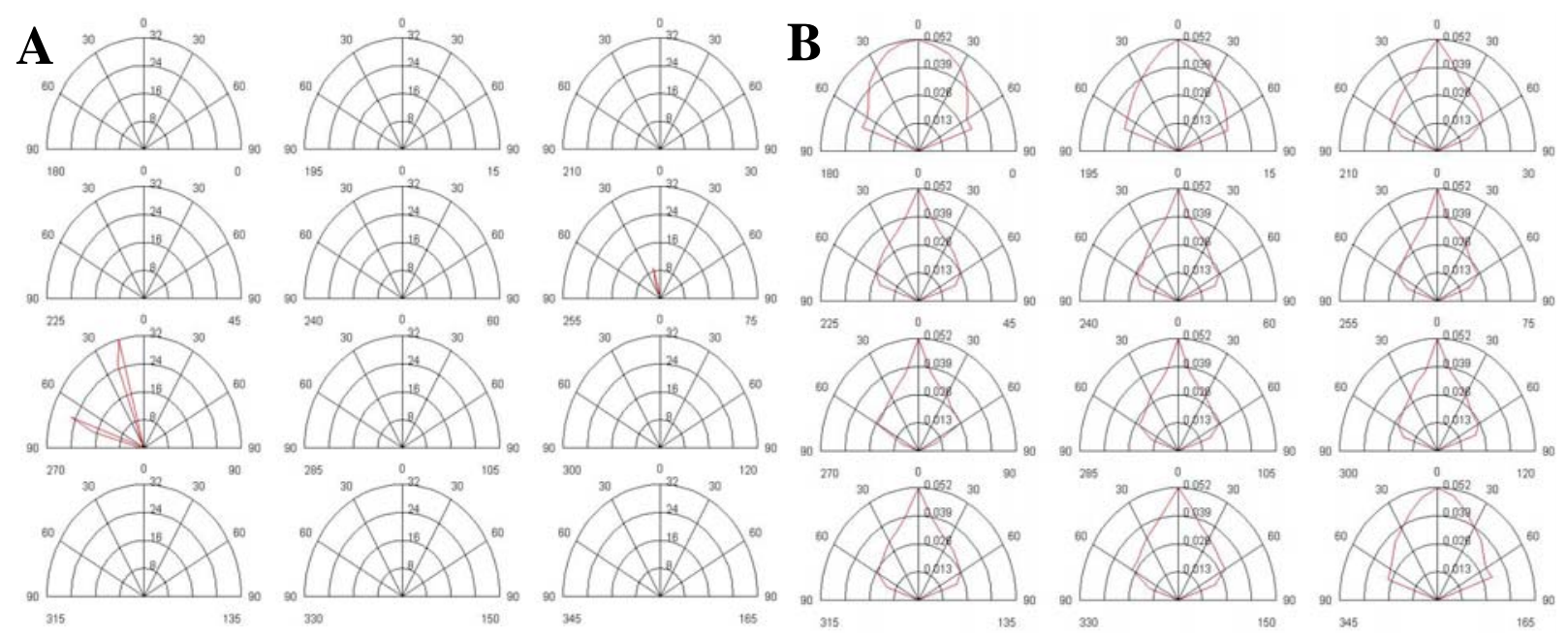

Fig. 8. Section views along C planes, given every $15^{\circ}$ in azimuth (i.e. planes C0-C180, C15-C195, etc., as can be read on the bottom of each graph): (A) Prismatic SOLF film (3M) : light incidence on flat glass side; $\left(\theta_{1}, \phi_{1}\right)$ $=\left(48^{\circ}, 90^{\circ}\right) ;\left(\Delta \theta_{2}, \Delta \phi_{2}\right)=\left(5^{\circ}, 5^{\circ}\right)$. One can observe the two narrow peaks on the C270 plane; (B) Pleated textile solar blind (Baumann-Hüppe AG) : $\left(\theta_{1}, \phi_{1}\right)=\left(0^{\circ}, 0^{\circ}\right) ;\left(\Delta \theta_{2}, \Delta \phi_{2}\right)=\left(10^{\circ}, 15^{\circ}\right)$. One can observe a general diffuse behaviour, with an enhancement of values along the plane $\mathrm{C} 0-\mathrm{C} 180$.

\section{Extraction of information from graphical representations}

As an example of reading the graphical representations, a pair of samples has been chosen to be discussed: a conventional white lamellae blind and an optimized solar blind, called "Shine", presenting a pearl grey quartz coating similar to the painting used in car body manufacturing and of particular shape (section similar to the profile of a spoon). Both blinds are manufactured by Baumann-Hüppe $A G$ and are shown in Fig. 9. To compare performances, the samples have been set with the equal slat inclinations ( $30^{\circ}$ with regard to the window plane) and dimensions (profile $10 \mathrm{~cm}$, length $34 \mathrm{~cm}$, distance between slats 8 $\mathrm{cm})$.
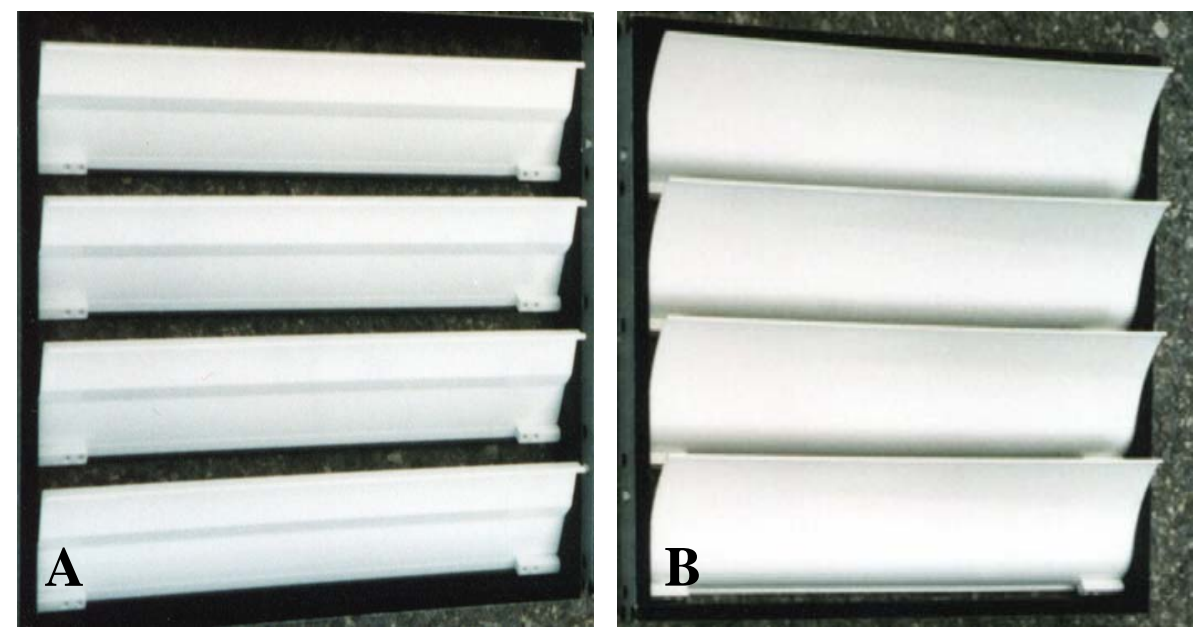

Fig. 9. Pictures of analysed venetian blinds: (A) conventional white lamellae; (B) optimized prototype "Shine”, with pearl grey quartz coating and a particular shape of slats. 
The observation of Figs. 10 and 11 shows important discrepancies between the transmission features of the conventional white blind and the "Shine" prototype under normal incidence (i.e. $\theta_{1}=0^{\circ}$ ).

The first one mainly presents a regular transmission through the slats (peak along the incident direction, pointed out by (a)), with a small effect of reflection on the coating, leading to a little increase of BTDF values in direction $\left(\theta_{2}, \phi_{2}\right)=\left(60^{\circ}, 90^{\circ}\right)$, pointed out on the hemispherical projection by (b).

The second one shows no direct component and presents a quasi-diffuse transmittance (with only a slight deformation of the photometric solid in favour of normal transmittance, (c)), except along a particular direction, which shows an important deviation of light due to reflection on the slats (pointed out by (d)).

Even though of same global design, the two venetian blinds can therefore present very different light-transmission features, only because their slats coating and profile are dissimilar. Under this incidence, the "Shine" prototype offers an important diffuse transmittance, and redirects light out of the direct transmittance direction, whereas the conventional blind only slightly affects the incident light path, and shows a quasi-negligible effect of diffuseness. It can be added that the observed BTDF extrema are seven times lower for the optimized blind than the light peak with conventional slats, leading to a diffuse redistribution that could be efficiently used to reduce glare risks under clear sky.
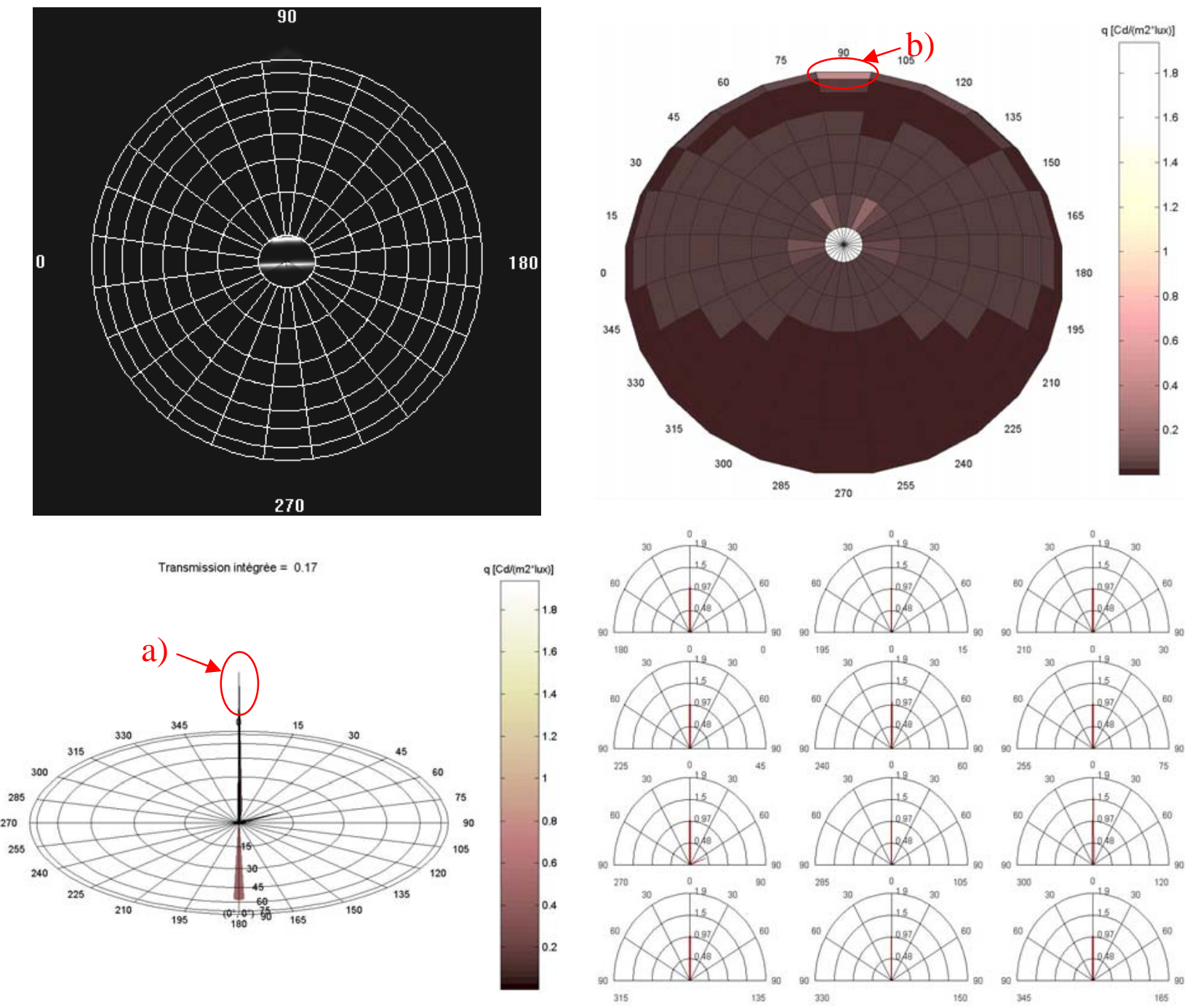

Fig. 10. White slats: Graphical representation of BTDF data under incidence $\left(\theta_{1}, \phi_{1}\right)=\left(0^{\circ}, 0^{\circ}\right)$. 

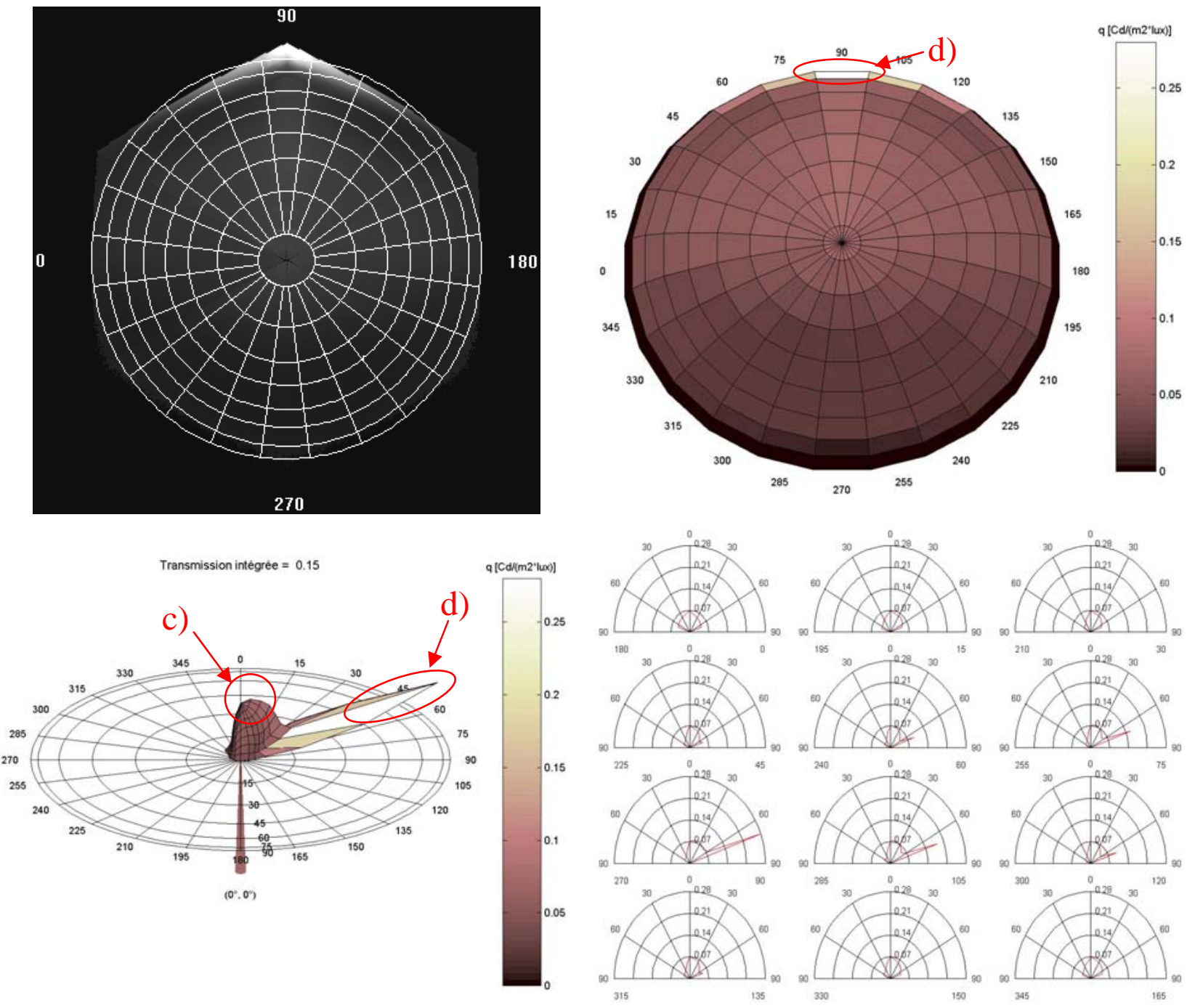

Fig. 11. "Shine”: Graphical representation of BTDF data under incidence $\left(\theta_{1}, \phi_{1}\right)=\left(0^{\circ}, 0^{\circ}\right)$.

The same features appear for larger incidence angles $\theta_{1}$ (Figs. 12 and 13): one can observe a regular transmission for the white slats, with small effects of reflection, displaced towards higher azimuths (anticlockwise on the projections, (e)). For the "Shine" prototype, the light transmission is quite diffuse, yet with a clearer deformation along the direct transmission direction (f)); this direction indeed gets closer to the reflected direction, which generates a superposition of effects.

For other angular incidences along the azimuthal plane C90 (i.e. $\phi_{1}=90^{\circ}$ ), the transmission features of both blinds are quite similar, as can be observed on Figs. 14 and 15: the major part of the incoming light is reflected on the slats and redirected along a coplanar direction (transmission peaks around $\phi_{2}=90^{\circ}$; cf. (g)); a minor part is slightly diffused, more significantly for the white slats (h), which also presents a more extended region for the reflection peak (cf. (i)).

Finally, along the azimuth plane C270 (i.e. $\phi_{1}=270^{\circ}$ ), the incoming light flux is almost not deviated, when passing through the blind types (j, k). Fig. 16 and 17 show that both blinds present almost the same regular transmitted components and even similar BTDF ranges. As these kinds of incidences are in fact never reached when considering the effective possible sun positions, such conclusions do not affect the appreciation of the products. 

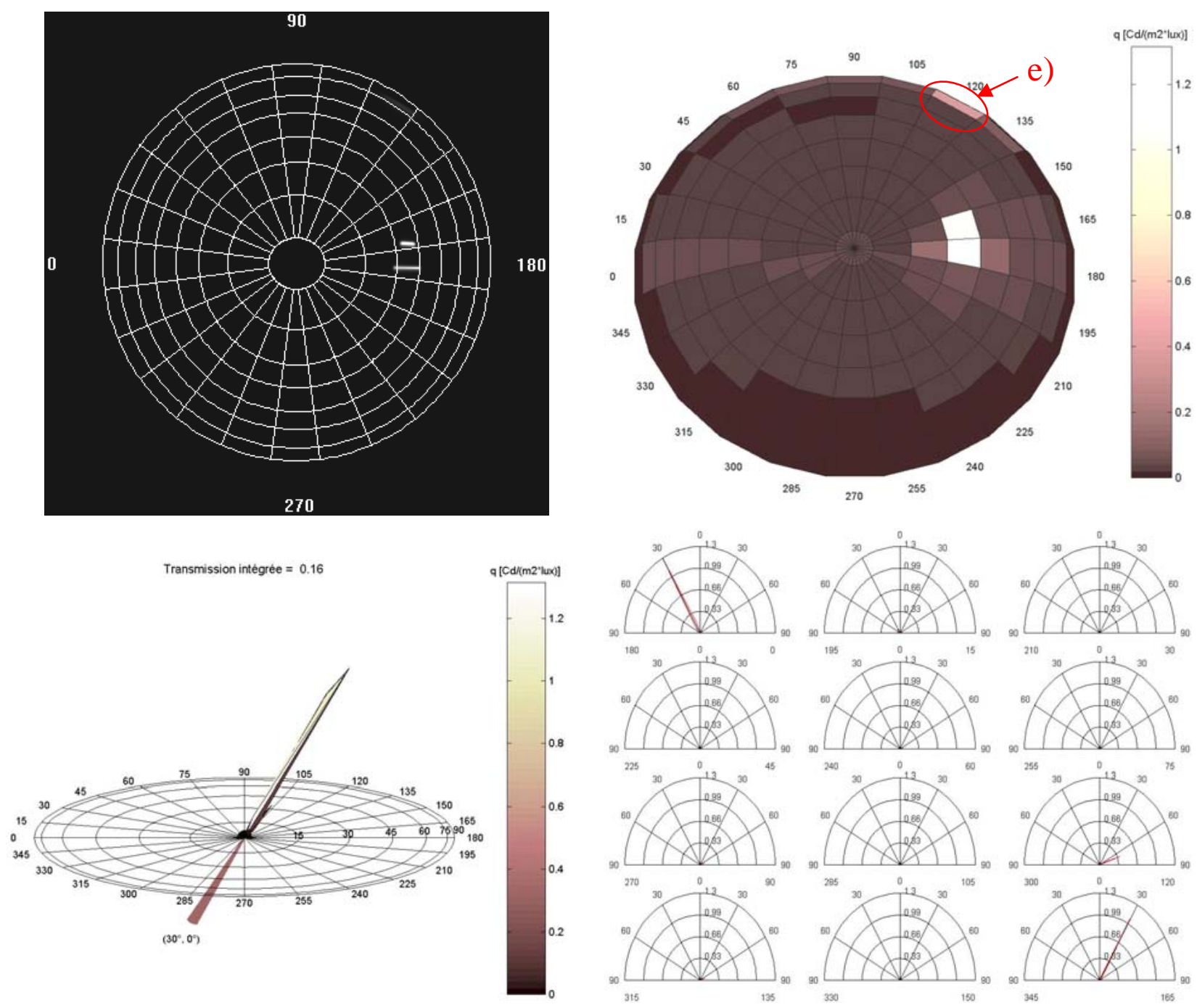

Fig. 12. White slats: Graphical representation of BTDF data under incidence $\left(\theta_{1}, \phi_{1}\right)=\left(30^{\circ}, 0^{\circ}\right)$.
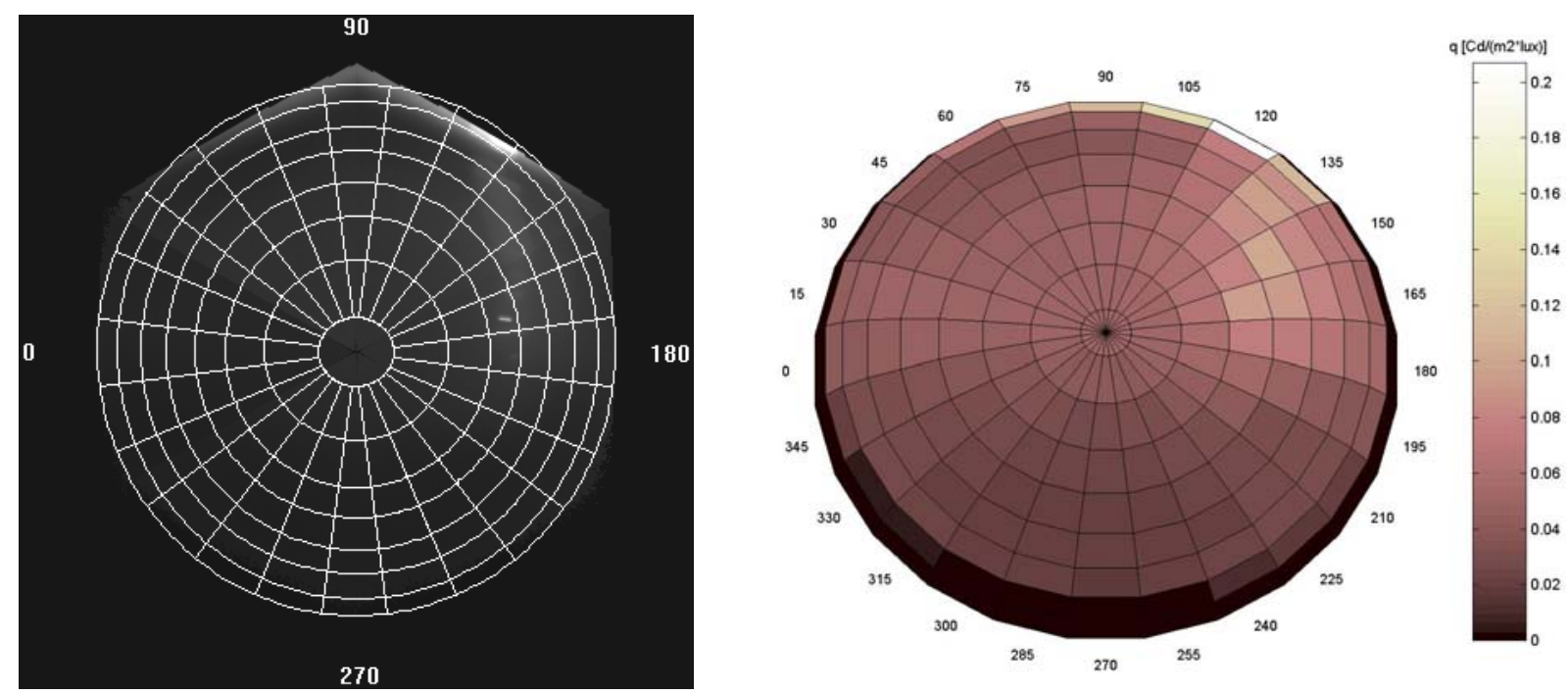

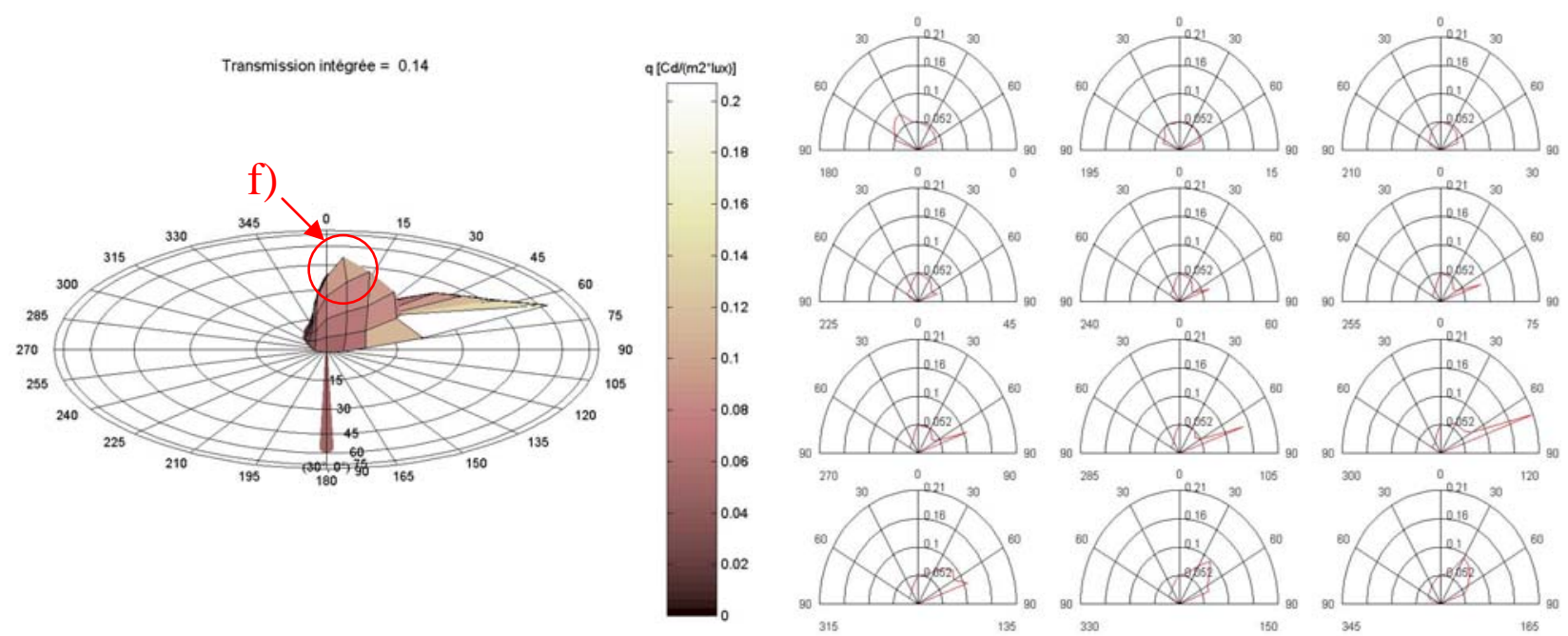

Fig. 13. "Shine”: Graphical representation of BTDF data under incidence $\left(\theta_{1}, \phi_{1}\right)=\left(30^{\circ}, 0^{\circ}\right)$.
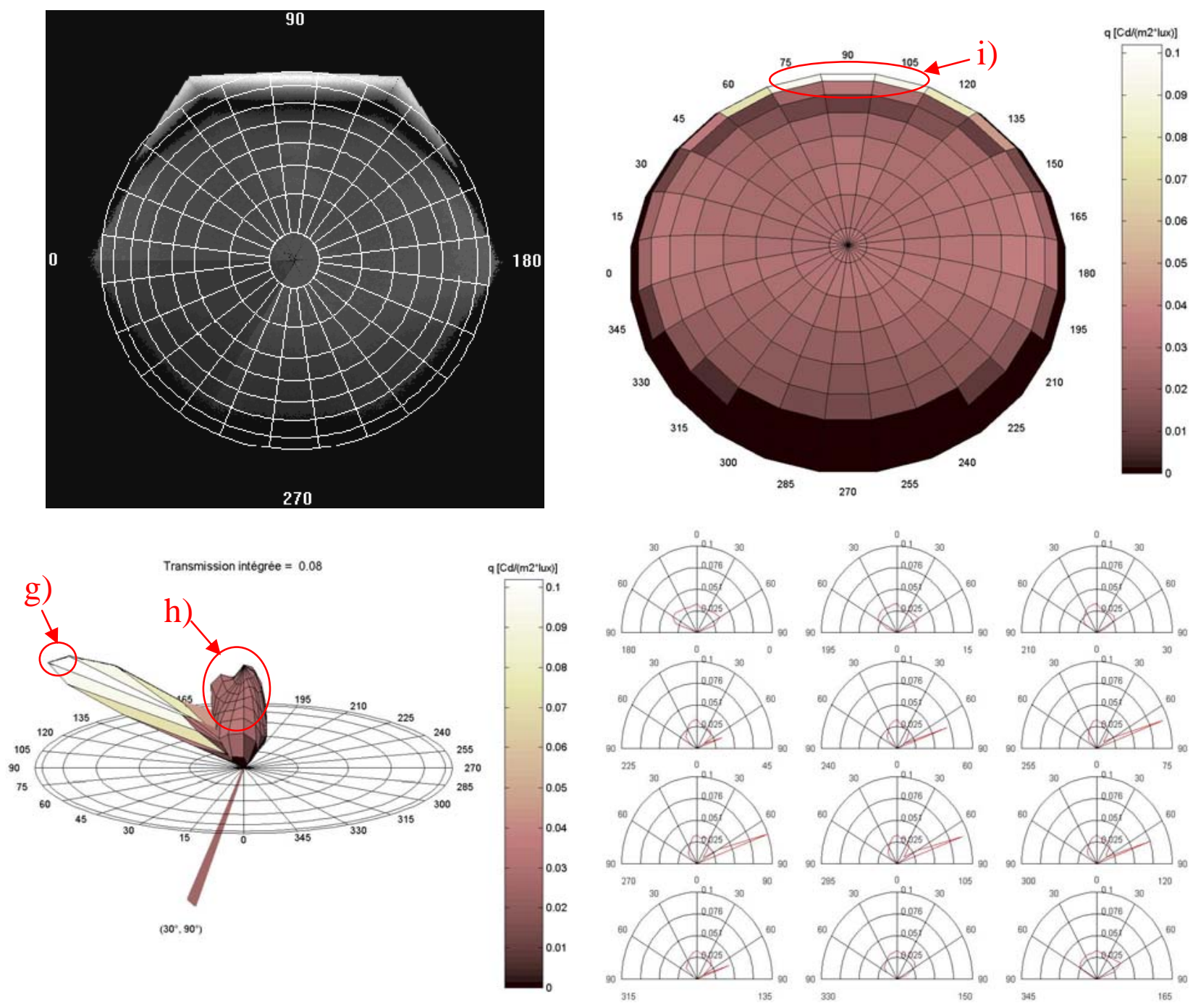

Fig. 14. White slats: Graphical representation of BTDF data under incidence $\left(\theta_{1}, \phi_{1}\right)=\left(30^{\circ}, 90^{\circ}\right)$. 

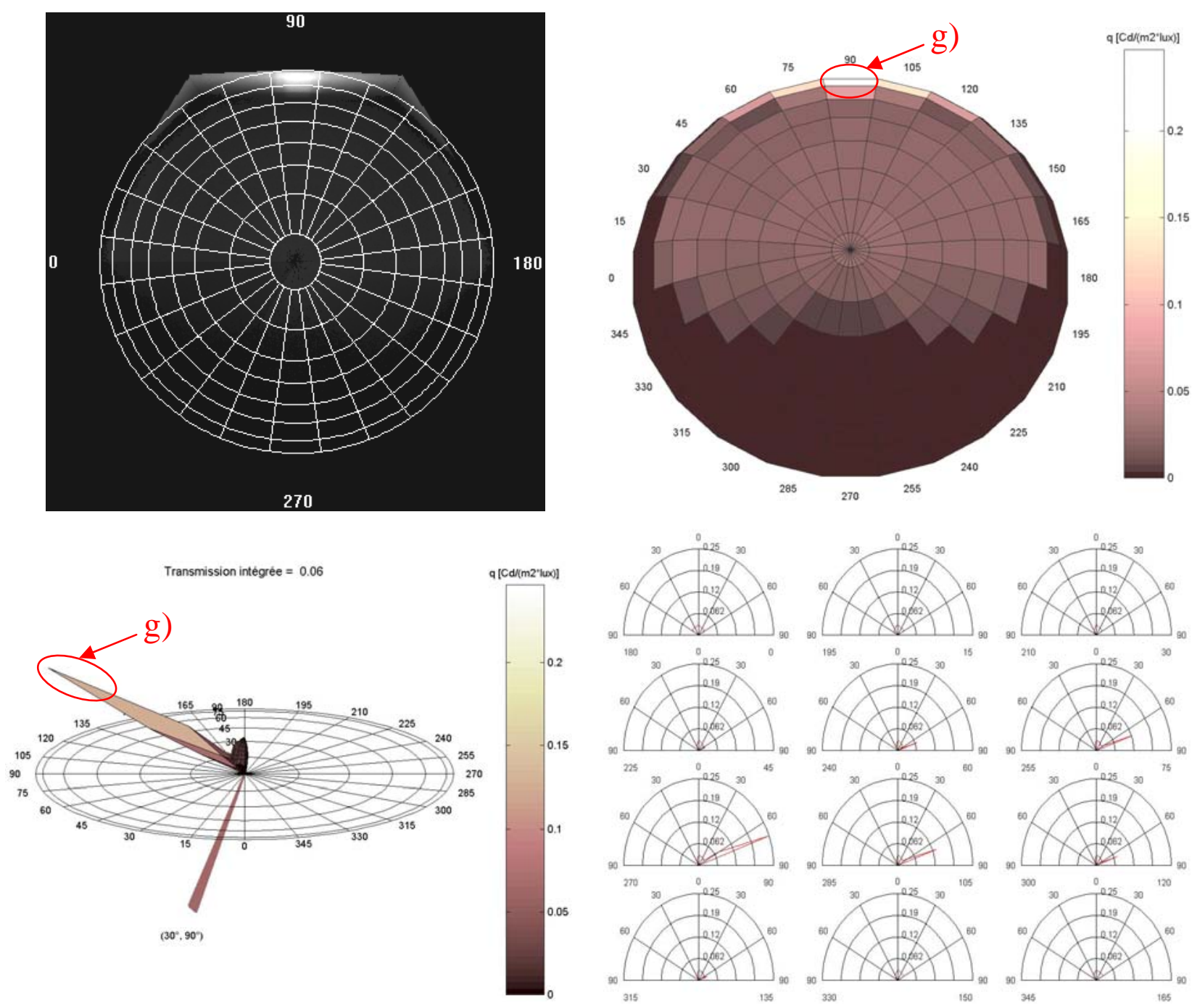

Fig. 15. "Shine": Graphical representation of BTDF data under incidence $\left(\theta_{1}, \phi_{1}\right)=\left(30^{\circ}, 90^{\circ}\right)$.
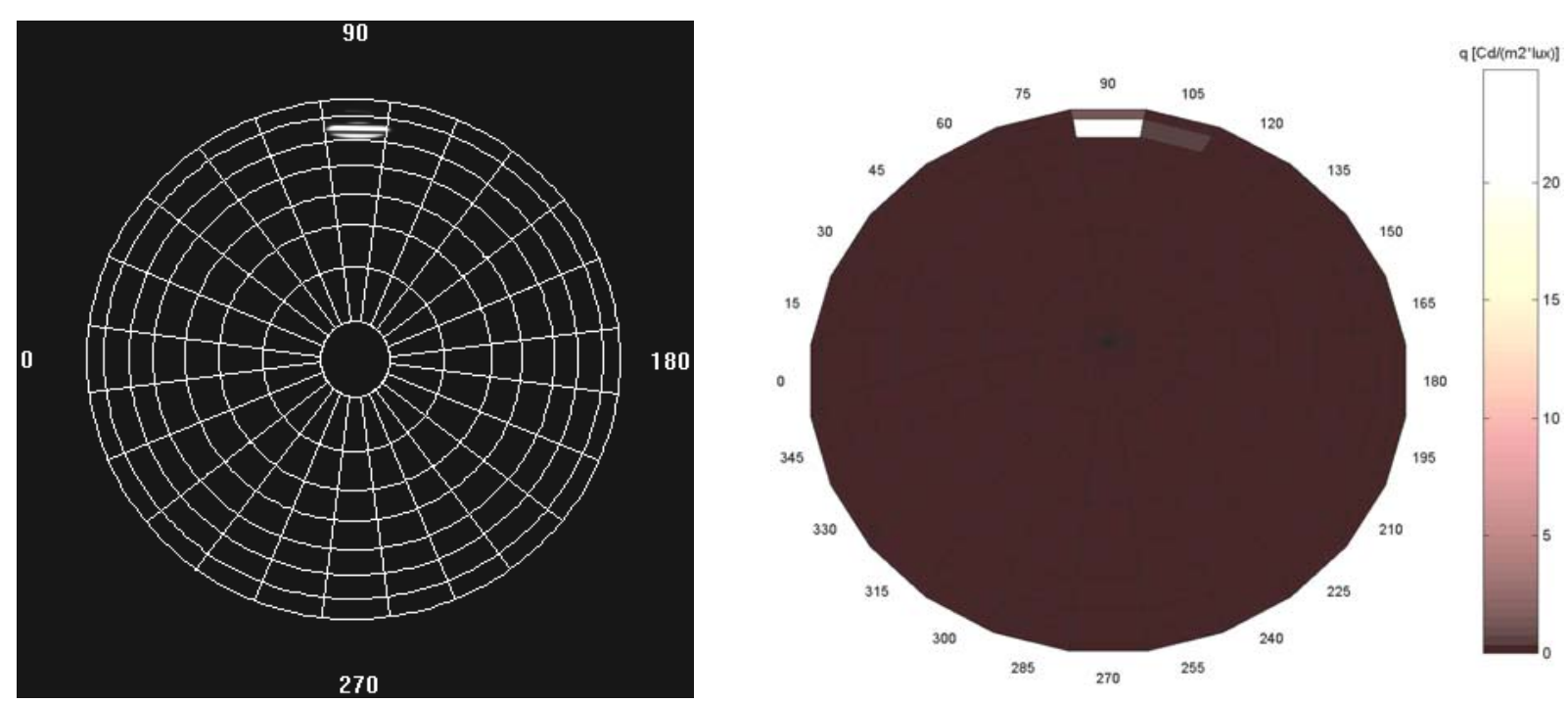

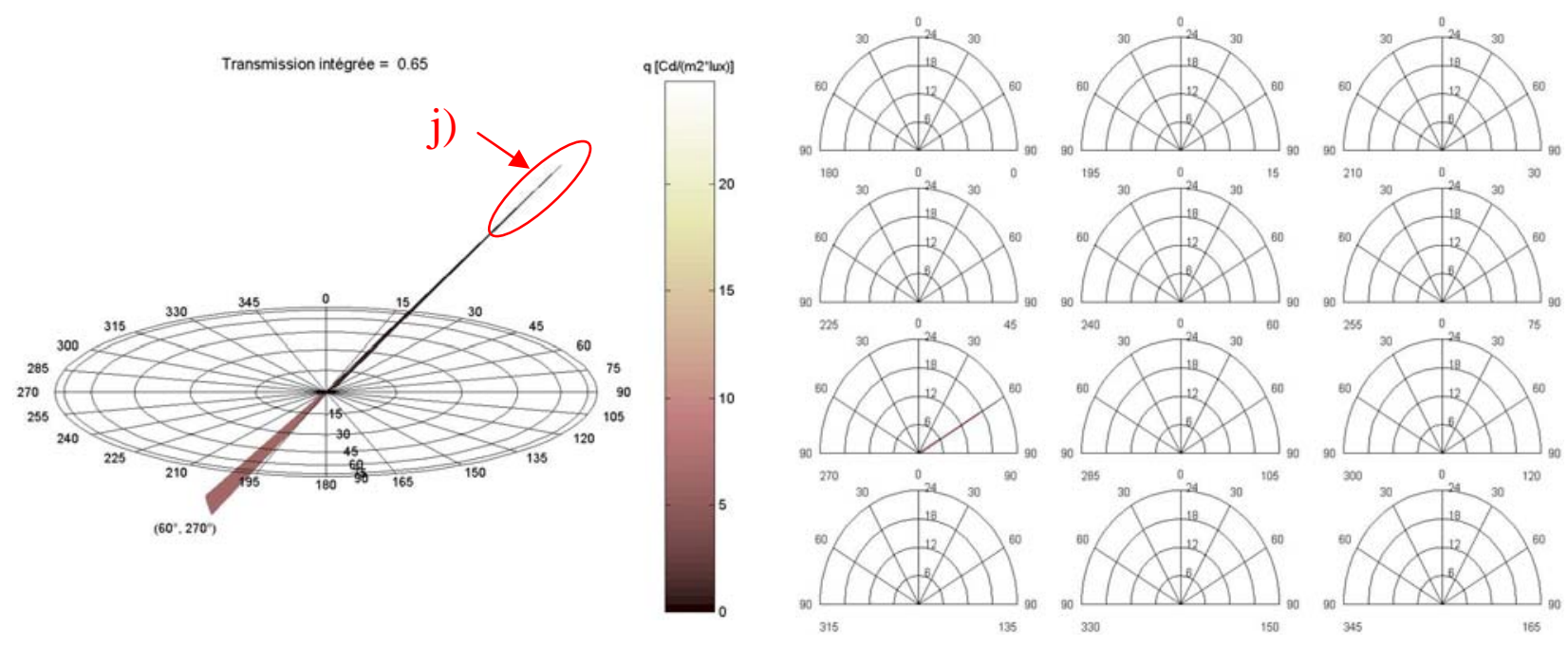

Fig. 16. White slats: Graphical representation of BTDF data under incidence $\left(\theta_{1}, \phi_{1}\right)=\left(60^{\circ}, 270^{\circ}\right)$.
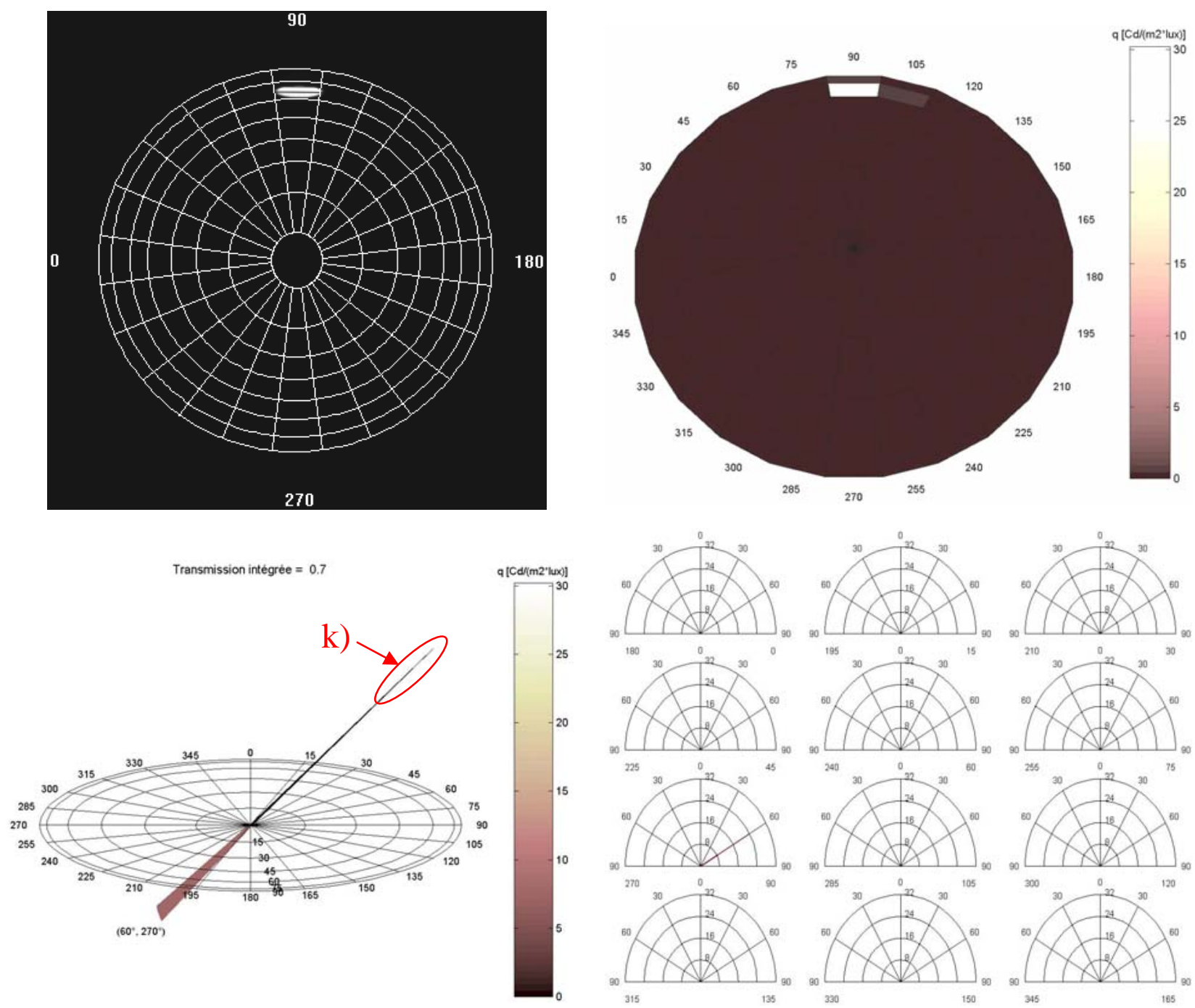

Fig. 17. "Shine”: Graphical representation of BTDF data under incidence $\left(\theta_{1}, \phi_{1}\right)=\left(60^{\circ}, 270^{\circ}\right)$. 
As we can see, the analysis of these figures leads to a good understanding of the transmission features of the characterized products. It can be deduced, for instance, that the "Shine" prototype can take advantage of its diffusing properties to reduce glare risks. Moreover, its significant redirecting (reflecting) capability can be used for many incident positions to illuminate the ceiling, which therefore becomes a secondary natural light source.

By combining the examination of the BTDF graphical representations with a detailed analysis of the numerical data contained in the final data file, a more thorough investigation of the products can be realized to formulate specific and quantitative conclusions concerning the slats shape or coating.

A simplified example of evaluation and comparison of visual comfort performances thanks to BTDF values is presented in the Appendix for the same solar blinds. In this "manual" example, unique incoming and emerging directions are chosen for simplicity. Of course, to realise a true prototype's optimization, the number of analysed directions (incident and transmitted) will be much larger and will in most cases require being integrated into a specific calculation programme to apply the results to various situations: different sun positions, system orientation, room dimensions, additional objects properties, and to assess the system's performances for precise issues: illuminance on a desk, proportion of work time where natural light is sufficient, glare situations, etc. For some applications, an averaging over a certain period (day, month, season, year) or over different room configurations can become useful.

All these applications require detailed information about the system's exact transmission properties, which are provided by its BTDF data set.

\section{Discussion}

As shown by the above example, having access to the BTDF values of a system allows outlining its positive, as well as its restricting characteristics. It also provides the necessary guidelines for practitioners to improve their systems, and offers an efficient way to clearly present product features to clients in the building construction sector.

By analysing the BTDF data and their representations, one can point out the suitable adjustments of shapes and/or coatings (reflection coefficients, texture, etc.), objectively determine the way such changes will affect the system's performances, which provides a very important potentiality for manufacturers to optimize their products. In some cases, it will be worthwhile submitting an optimized prototype designed according to the new specifications based on the BTDF data examination to a new characterization, in order to determine whether the reached performances are acceptable or still to be perfected.

Moreover, by analysing BTDF data sets, architects can judiciously select the fenestration systems for their particular project, basing their choice on objective and precise criteria. Note too that integrating BTDF data into daylight simulation tools has become essential for an accurate calculation of the light propagation inside a given room.

Of course, only some incident directions have been presented in this paper, even though an effective product characterization consists of a much larger set of incidences.

A default set of incident directions has been determined within the framework of Task 21 of the International Energy Agency (Aydinli, 1999), that conforms the sky luminance distribution defined by Tregenza (1987) and which is therefore composed of 145 distinct directions distributed over the incident hemisphere according to regular solid angles intervals. An alternative investigation possibility would be to follow the solar course for a particular 
building location and façade orientation to get a detailed characterization under realistic conditions.

In fact, a fully automated characterization can be realized by the photogoniometer piloting software with any kind of incident directions set (Andersen et al., 2000), whether according to all or part of the 145 Tregenza directions, to a regular grid, along given $\mathrm{C}$ planes, or according to any set of freely chosen angular couples $\left(\theta_{1}, \phi_{1}\right)$.

Another important parameter that can be adjusted to the user's or practitioner's needs is the output resolution $\left(\Delta \theta_{2}, \Delta \phi_{2}\right)$, which allows to create a BTDF data set specifically adapted to the kind of information that will be extracted from it: global light distribution inside a room, narrow glare problems, etc.

\section{Conclusion}

The development of advanced fenestration systems (new solar blinds, complex glazing materials, daylight redirecting devices) can significantly contribute to reduce energy consumption of buildings, and can simultaneously improve visual comfort conditions for users. However, a full knowledge of the light distribution through a window, especially if it is complex, is necessary to optimize its performance, e.g. to deepen the propagation of daylight into the room or to increase solar gains in winter and decrease solar loads in summer. Moreover, detailed information about the fenestration system's transmission properties is also required for daylight simulation tools to base the calculations on true windows luminous characteristics. The latter can be formulated physically in terms of BTDFs, which are assessed by a bi-directional photogoniometer, the one presented here being based on digitalimaging techniques, to accelerate considerably BTDF measurements as well as to provide a continuous and accurate information about the transmitted light distribution, even when high luminance gradients appear.

Once a given window system has been characterized according to its BTDF, the way it transmits and redistributes the light inside a room is precisely known, and it can be used in different ways: to facilitate and optimize the conception, the development and the manufacturing of advanced fenestration materials, as well as their judicious selection, to increase the performances of daylighting simulation programmes used in building design, and, as a further step, in order to allow a categorization of these materials based on a sufficiently large BTDF database, for their market distribution.

A special attention has been paid to the data processing to provide an easy and synthetic approach of the light transmission properties of the materials. Different graphical representations have been created with appropriately developed software in the form of photometric solids of three different kinds (hemispherical projection, axonometric view, Cplanes cross-cutting), built in spherical coordinates for an intuitive representation of the lighttransmission features.

\section{Acknowledgements}

This work was supported by the Swiss Federal Institute of Technology (EPFL) and the Commission for Technology and Innovation (CTI). The author thanks Dr. Laurent Michel for preliminary work on graphical BTDF representation. 


\section{References}

Andersen, M., Michel, L., Roecker, C., Scartezzini, J.-L. (2001) Experimental assessment of bi-directional transmission distribution functions using digital imaging techniques. Energy and Buildings 33(5), 417-431.

Andersen, M., Scartezzini, J.-L., Roecker, C., Michel, L. (2000) Bi-directional Photogoniometer for the Assessment of the Luminous Properties of Fenestration Systems. CTI Project No. 3661.2, LESO-PB/EPFL (ed.), Lausanne.

Aydinli, S. (1999) Report on physical quantities to be Measured in Laboratory Facilities. IEASHC Task 21, TUB (ed.), Berlin.

Commission Internationale de l'Eclairage (1977) Radiometric and photometric characteristics of materials and their measurement. CIE 38 (TC-2.3) $2^{\text {nd }}$ Edition, Paris.

Illuminating Engineering Society of North America (1984) Lighting Handbook - Application and Reference Volume. IESNA, New York.

Office Fédéral des Questions Conjoncturelles (1994) Eclairage des bureaux - Programme d'action RAVEL - Utilisation rationnelle de l'électricité. OCFIM 724.329.2f, Bern.

Scartezzini, J.-L. and Paule, B. (1994) Office Lighting. EDMZ (ed.), Bern.

Tregenza P.R. (1987) Subdivision of the sky hemisphere for luminance measurements. Lighting Research and Technology 19, CIBSE.

Wirth, H. (1999) Richtungsselektive Steuerung von Sonnenstrahlung durch Fenster. PhD Thesis, EPFL (ed.), Lausanne.

\section{Appendix: Analysis example of visual comfort performance based on BTDF data}

On the basis of the BTDF data set of a given fenestration system, it is possible to deduce whether the transmitted luminances will induce glare effects or not, and whether daylight will be sufficient for the kind of work considered. As shown by equation (2), the luminances produced through a given blind in each direction can be directly deduced from the BTDF values associated with these directions, assuming that the illuminance $E_{1}$ is known. A possible $E_{1}$, for example, would be the illuminance on a vertical plane measured under clear sky for a particular sun position when studying frontal openings.

As prescribed by the lighting recommendations, a situation is considered as comfortable for the human eye if the luminance contrasts do not exceed the ratio 1:3 in the ergorama, limited by $30^{\circ}$ of angular opening, and 1:10 in the panorama (OFQC, 1994), limited by $60^{\circ}$ of angular opening (Fig. A1). 


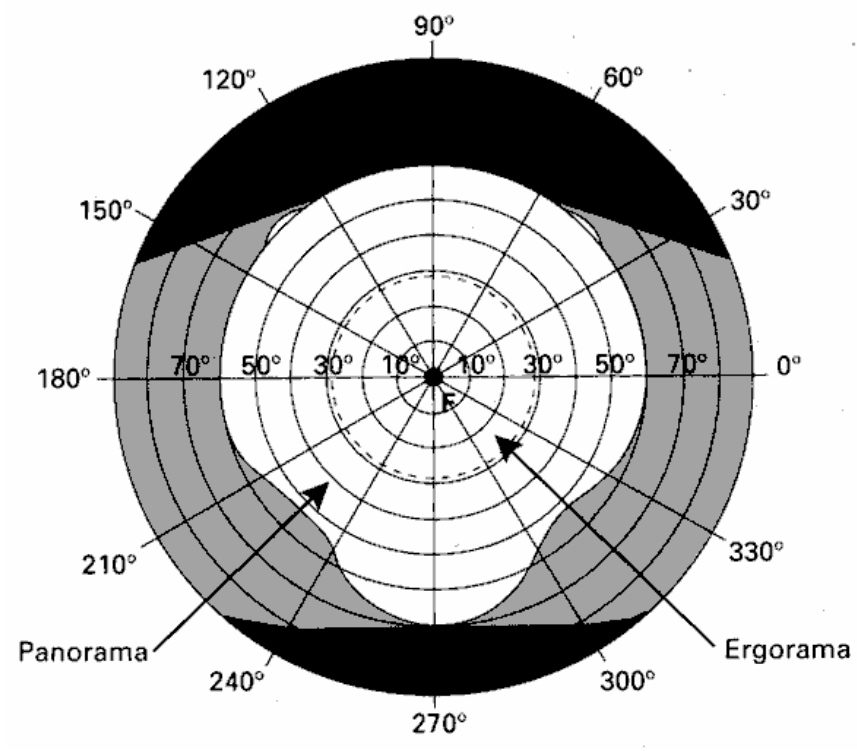

Fig. A1. Human field of vision (IESNA, 1984): white, field simultaneously seen by both eyes; grey, field seen by one eye; $\mathrm{F}$, central field (fovea).

Consider the situation of a computer workplace near a window on which a solar blind is installed, oriented perpendicularly to the wall. This situation is shown by Fig. A2.

We consider the window as of same elevation as the worker's eyes, and examine the BTDF values associated to the outgoing angular couple $\left(\theta_{2}, \phi_{2}\right)=\left(0^{\circ}, 0^{\circ}\right)$, under a fictive sunlight incident direction $\left(\theta_{1}, \phi_{1}\right)=\left(0^{\circ}, 0^{\circ}\right)$ : $\operatorname{BTDF}\left(0^{\circ}, 0^{\circ}, 0^{\circ}, 0^{\circ}\right)=1.937\left[\mathrm{~cd}^{\circ} \mathrm{m}^{-2} \cdot \mathrm{lx}^{-1}\right]$ for the white slats and to 0.070 [cd $\left.\cdot \mathrm{m}^{-2} \cdot \mathrm{lx}^{-1}\right]$ for the "Shine" prototype. Of course, a generalization of this example to other directions $\left(\theta_{2}, \phi_{2}\right)$ (and other incidences $\left(\theta_{1}, \phi_{1}\right)$ ) would be necessary for assessing real situations.

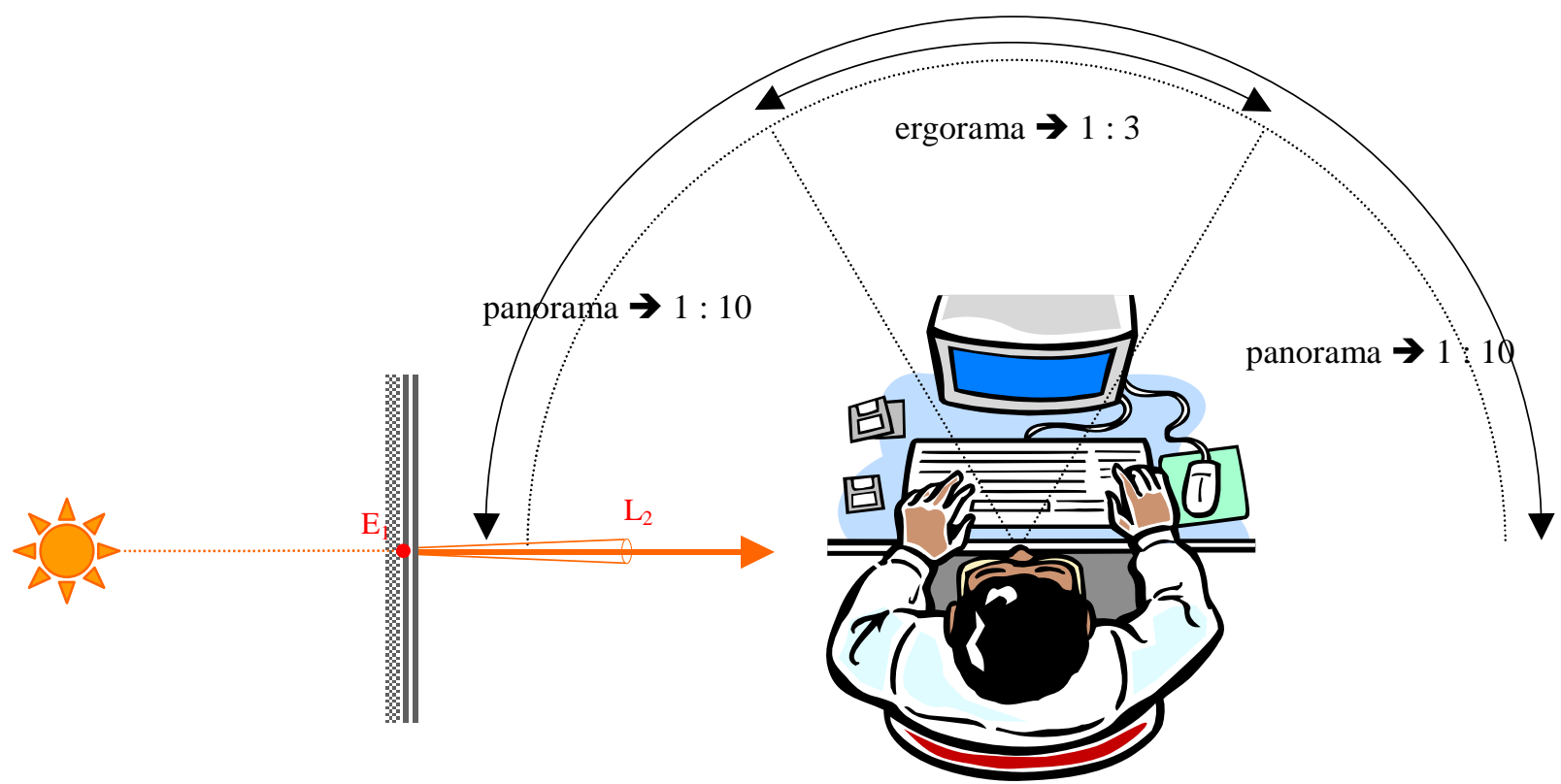

Fig. A2. Vertical view of a working place situated nearby a frontal window, with the subdivision of the field of vision into ergorama and panorama, together with the acceptable luminance contrasts associated. The panorama is extended for sake of simplicity to a $180^{\circ}$ opening angle. 
To account for the reduction of the daylight flux by the window glazing (situated behind the blinds), each BTDF value is multiplied by an approximate transmission factor 0.85 (double glazing), which leads to respective values of 1.646 and 0.060 for the two blinds. An illuminance $E_{1}=40000 \mathrm{~lx}$ has been chosen as being reasonably representative of clear sky conditions, and the emerging luminances $\mathrm{L}_{2}$ can, therefore, be calculated according to equation (2): we have $\mathrm{L}_{2}$ white slats $=65858\left[\mathrm{~cd}^{\prime} \mathrm{m}^{-2}\right]$ and $\mathrm{L}_{2}$ "Shine" $=2380\left[\mathrm{~cd} \cdot \mathrm{m}^{-2}\right]$.

As the luminance produced by a computer screen is in general comprised between 100 and 200 [cd $\cdot \mathrm{m}^{-2}$ ], one can deduce that emerging luminances $>2000$ [cd $\mathrm{m}^{-2}$ ] will probably produce glare effects for the worker, the luminance contrast then being $>10$. In our case study, one immediately sees that the "Shine" prototype will induce a far more comfortable situation than the conventional white slats, where direct transmission is very important (Fig. 10).

We also know from the graphical representations presented in Figs. 11, 13 and 15 that the major part of a beam reaching the "Shine" prototype is transmitted along a grazing direction $\left(\theta_{2}=70^{\circ}\right.$ : reflection on the slats), which shows that glare will probably not occur at a reasonable distance from the window.

The purpose of this rudimentary study is to show a way to extract information from BTDF data sets, and to point out the complementarity and usefulness of both graphical and numerical forms. As mentioned before, supplementary measurements and analyses (multiple incident and emerging directions, different sample orientations and slats inclinations, various positions for the worker and the window, etc.) are necessary to achieve a reliable expertise of the photometric characteristics of a system. It can be noted that the use of computational methods can often become practical or necessary, as for the assessment of daylight performances inside a room (e.g. illuminance at a given position), where a lot of factors must be taken into account (dimensions, position, reflection factor of components, etc.). 